\title{
A multi-parameter criterion for layer formation in a stratified shear flow using sorted buoyancy coordinates
}

\author{
J. R. T A Y L O R $\mathbf{R}^{1} \dagger$ Q. $\mathrm{ZHOU}^{1}$ \\ ${ }^{1}$ Department of Applied Mathematics and Theoretical Physics, \\ University of Cambridge, Centre for Mathematical Sciences, Wilberforce Road, CB3 0WA, \\ Cambridge
}

(Received ?; revised ?; accepted ?. - To be entered by editorial office)

Here, we examine the conditions for layer formation in a stratified shear flow. We reformulate the conditions for amplification of small perturbations to a uniform stratification first proposed by Phillips (1972) and Posmentier (1977) using the sorted buoyancy coordinates introduced by Winters \& D'Asaro (1996) and Nakamura (1996). We consider the possible dependence of the effective diffusivity on three nondimensional parameters: the gradient Richardson number, the buoyancy Reynolds number, and the Prandtl number, and obtain a simple expression for conditions favourable for layer formation. The new framework is applied to direct numerical simulations of stratified shear flow. We then apply a recent multi-parameter parameterization developed by Salehipour et al. (2016), which suggests that layer formation is favoured for large Prandtl numbers and moderate to large values of the gradient Richardson and buoyancy Reynolds numbers.

\section{Introduction}

Fine-scale vertical profiles of density in the ocean and atmosphere sometimes take the appearance of a series of step-like layers of nearly uniform density separated by strongly stratified interfaces. Several explanations for the formation of these layers have been proposed, including double-diffusion (Schmitt 1994; Radko 2016), localized breaking of internal gravity waves (Garrett \& Munk 1971), shear instabilites (Pelegrí \& Sangrà 1998) and amplification of small disturbances to a linear density profile (Phillips 1972).

Phillips (1972) proposed a simple thought experiment to illustrate how layers could develop from small perturbations to a uniformly stratified fluid. He showed that if the magnitude of the buoyancy flux is a decreasing function of the local gradient Richardson number, $J \equiv N^{2} / S^{2}$, where $N \equiv \sqrt{\partial b / \partial z}$ is the buoyancy frequency and $S \equiv \partial u / \partial z$ is the vertical derivative of the horizontal velocity, small disturbances to the initial stratification profile can grow in time. Using a parameterized form of the buoyancy flux, Posmentier (1977) showed that this mechanism can lead to the formation of distinct step-like layers.

In subsequent years, laboratory experiments were conducted which appeared to support the idea of the emergence of layers in forced stratified turbulence. These experiments have recently been revisited by Thorpe $(2016)$. Linden $(1979,1980)$ found evidence in various experimental configurations that the flux Richardson number, $R_{f}$, defined as the fraction of the available kinetic energy which induces a change in the potential energy by mixing the density profile, can be a non-monotonic function of the overall (or bulk)

$\dagger$ Email address for correspondence: J.R.Taylor@damtp.cam.ac.uk 
Richardson number, $J_{o}$. At small values, $R_{f}$ increased with increasing $J_{o}$, while for values of the overall Richardson number larger than a critical value, $J_{c}$, the flux Richardson number decreased with increasing values of $J_{o}$. For a constant rate of work on the fluid, $R_{f}$ is proportional to the buoyancy flux. Therefore, decreasing $R_{f}$ with increasing $J_{o}$ for $J_{o}>J_{c}$ is consistent with the layering mechanism discussed by Phillips (1972) and Posmentier (1977).

Park et al. (1994) conducted laboratory experiments where a salt-stratified fluid with an initially linear buoyancy profile was stirred with an oscillating vertical rod. They found that the buoyancy profile develops a series of step-like layers when the overall Richardson number is sufficiently large and the Reynolds number is sufficiently small. They found that the critical Richardson number separating the layering and non-layering regimes was $J_{c} \approx \exp (\operatorname{Re} / 900)$. The dependence of the flux Richardson number was generally consistent with the Phillips (1972) and Posmentier (1977) mechanism for layer formation.

Accumulating evidence from numerical simulations and laboratory experiments indicates that stratified turbulence depends on at least one more non-dimensional parameter, $G \equiv \varepsilon /\left(\nu N^{2}\right)$, where $\varepsilon$ is the dissipation rate of turbulent kinetic energy and $\nu$ is the kinematic viscosity (Shih et al. 2005; Brethouwer et al. 2007; Lozovatsky \& Fernando 2013; Bouffard \& Boegman 2013; Bartello \& Tobias 2013; Mater \& Venayagamoorthy 2014a,b; Portwood et al. 2016) †. This nondimensional number is sometimes called the buoyancy Reynolds number since it can be formed using the velocity scale $U=\varepsilon^{1 / 3} L_{O}^{1 / 3}$ and the Ozmidov scale, $L_{O} \equiv \varepsilon^{1 / 2} N^{-3 / 2}$ (Dillon \& Caldwell 1980). By definition, $G \equiv\left(L_{O} / L_{K}\right)^{4 / 3}$, where $L_{K} \equiv \nu^{3 / 4} \varepsilon^{-1 / 4}$ is the Kolmogorov scale and $G$ can be interpreted as an indication of the scale separation between the largest turbulent overturns (proportional to the Ozmidov scale) and the smallest motions allowed by viscosity (proportional to the Kolmogorov scale) (Gargett et al. 1984). For reference, a collection of observations indicates that the buoyancy Reynolds number is typically $\mathrm{O}(1-1000)$ in the ocean (Salehipour et al. 2016).

Here, we focus on layer formation in shear-driven stratified turbulence. Motivated by this and the recent parameterization developed by Salehipour et al. (2016), we will emphasize the role of the gradient Richardson, buoyancy Reynolds, and Prandtl numbers on the condition for layer formation. As noted by Mater \& Venayagamoorthy $(2014 a, b)$, other non-dimensional parameters, such as the turbulent Froude number, might also be important. The relationship between the various nondimensional parameters characterizing stratified turbulence is still the topic of active research. As will be discussed in section 5, the analysis carried out here could also be extended to examine the roles of other nondimensional parameters on layer formation. Additionally, the nondimensional parameters are generally defined in terms of averaged quantities, and there is some ambiguity in how the average is defined. The particular choice used here will be defined explicitly in section 2 .

Below, in section 2, we extend the work of Phillips (1972) and Posmentier (1977) by invoking the tracer coordinates introduced by Winters \& D'Asaro (1996) and Nakamura (1996). This has the advantage of eliminating the reversible component of the buoyancy flux and generalizing the condition for layer formation into a simple expression. We also extend the analysis to explicitly include the buoyancy Reynolds and Prandtl numbers. In section 3, we apply the new framework to direct numerical simulations (DNS) of stratified shear flow. In section 4 we consider various functional forms for the effective diffusivity,

$\dagger$ Here, we use the symbol $G$ to recognize its early use by Gibson (1980) and Gargett et al. (1984) 
including a recent parameterization introduced by Salehipour et al. (2016) and examine when layer formation might occur.

\section{Formulation}

Winters \& D'Asaro (1996) and Nakamura (1996) derived an evolution equation for a tracer in sorted buoyancy coordinates. $\dagger$ Written here in terms of buoyancy, $b$, and sorted height, $z_{*}$, they showed that the buoyancy conservation equation in a closed system can be written

$$
\frac{\partial b}{\partial t}=\frac{\partial}{\partial z_{*}}\left(\kappa_{e} \frac{\partial b}{\partial z_{*}}\right)
$$

where

$$
\kappa_{e}=\kappa\left(\frac{\partial z_{*}}{\partial b}\right)^{2}\left\langle|\nabla b|^{2}\right\rangle_{z_{*}},
$$

$\kappa$ is the constant molecular diffusivity, $\nabla$ denotes the gradient vector in physical coordinates, and $\langle\cdot\rangle_{z_{*}}$ indicates an average over all points with the same value of $z_{*}$. The effective diffusivity can also be written $\kappa_{e}=\kappa\left(A_{S} / A\right)^{2}$, where $A_{S}$ is an area associated with distorted buoyancy surfaces, and $A$ is the area of an undisturbed (flat) buoyancy surface (Winters \& D'Asaro 1996). A simple interpretation is then that the effective diffusivity is larger for more highly distorted buoyancy surfaces with larger surface area, and is equal to the molecular diffusivity for undisturbed buoyancy surfaces (i.e. when the sorted profile is the same as the profile in physical coordinates.)

Taking a derivative of Eq. 2.1 with respect to $z_{*}$ gives an equation for the stratification in sorted height coordinates

$$
\frac{\partial N_{*}^{2}}{\partial t}=\frac{\partial^{2} \kappa_{e}}{\partial z_{*}^{2}} N_{*}^{2}+2 \frac{\partial \kappa_{e}}{\partial z_{*}} \frac{\partial N_{*}^{2}}{\partial z_{*}}+\kappa_{e} \frac{\partial^{2} N_{*}^{2}}{\partial z_{*}^{2}}
$$

where $N_{*}^{2} \equiv \partial b / \partial z_{*}$ is the buoyancy gradient in sorted height coordinates. Eq. 2.3 was recently used by Zhou et al. (2017) to analyze the evolution of a density interface in direct numerical simulations of stratified plane Couette flow. We will show below that for a nearly uniform stratification, the second term on the right hand side of Eq. 2.3 is expected to be small. In this case, the last term on the right hand side of Eq. 2.3 represents diffusion of the sorted buoyancy profile through combined molecular and turbulent diffusivity. Since $\kappa_{e} \geqslant \kappa>0$, the corresponding diffusivity is always positive. Therefore, variations in $N_{*}^{2}$ will grow in time when $\partial^{2} \kappa_{e} / \partial z_{*}^{2}>0$ and when the first term on the right hand side of Eq. 2.3 is large enough to overcome the effects of diffusion.

We will assume that changes in $\kappa_{e}$ with respect to the sorted height, $z_{*}$, and time, $t$, can be parameterized in terms of the gradient Richardson number, $J_{*}$, and buoyancy Reynolds number, $G_{*}$, defined in sorted height coordinates. Specifically,

$$
J_{*}\left(z_{*}, t\right) \equiv \frac{N_{*}^{2}}{S_{*}^{2}}, \quad G_{*}\left(z_{*}, t\right) \equiv \frac{\varepsilon_{*}}{\nu N_{*}^{2}},
$$

where $S_{*} \equiv\langle\partial u / \partial z\rangle_{z_{*}}$ and the kinetic energy dissipation rate is $\varepsilon_{*} \equiv 2 \nu\left\langle s_{i j} s_{i j}\right\rangle_{z_{*}}$, and $s_{i j}$ is the rate of strain tensor. In this definition, $s_{i j}$ is based on velocity gradients in physical space and the tensor product $s_{i j} s_{i j}$ is averaged along levels of constant sorted height. Note that the average operator used here is different than the more conventional

$\dagger$ Nakamura (1996) used a two-dimensional area coordinate which is equivalent to the sorted height coordinate used by Winters \& D'Asaro (1996) for a two-dimensional tracer in a domain with fixed horizontal extent. 
choice to average over constant unsorted height. We can also include dependence of $\kappa_{e}$ on the Prandtl number, $\operatorname{Pr} \equiv \nu / \kappa$, or any other constant parameter, i.e.

$$
\kappa_{e}=\kappa_{e}\left(J_{*}\left(z_{*}, t\right), G_{*}\left(z_{*}, t\right), \operatorname{Pr}, \ldots\right) .
$$

Since $\operatorname{Pr}$ is constant, derivatives of $\kappa_{e}$ with respect to $z_{*}$ involve variations with respect to $J_{*}$ and $G_{*}$, but not $\operatorname{Pr}$ directly.

Following the thought experiment proposed by Phillips (1972), consider the evolution of a stratified flow with a nearly uniform stratification and shear. Phillips (1972) further defined a turbulent velocity scale, $u_{*}=\sqrt{\tau / \rho_{0}}$, where $\tau$ is a constant stress and $\rho_{0}$ is the reference density. Here, we will instead assume that the turbulent kinetic energy dissipation rate, $\varepsilon_{*}$, is nearly constant. Let $N_{0}, S_{0}$, and $\varepsilon_{0}$ denote the constant background buoyancy frequency, shear, and dissipation rate. The buoyancy gradient, shear, and dissipation rate can be written in terms of small departures from the background values:

$$
\begin{gathered}
N_{*}^{2}\left(z_{*}, t\right)=N_{0}^{2}+\epsilon N_{1}^{2}\left(z_{*}, t\right)+O\left(\epsilon^{2}\right), \\
S_{*}\left(z_{*}, t\right)=S_{0}+\epsilon S_{1}\left(z_{*}, t\right)+O\left(\epsilon^{2}\right), \\
\varepsilon_{*}\left(z_{*}, t\right)=\varepsilon_{0}+\epsilon \varepsilon_{1}\left(z_{*}, t\right)+O\left(\epsilon^{2}\right),
\end{gathered}
$$

where $\epsilon$ is a small expansion parameter. Here all quantities are defined in sorted buoyancy coordinates, but the $*$ subscript has been omitted from $N_{0}, N_{1}, S_{0}$, etc. to simplify the notation.

To leading order in $\epsilon$, derivates of the gradient Richardson number and buoyancy Reynolds number are

$$
\frac{\partial J_{*}}{\partial z_{*}}=\epsilon\left[\frac{1}{S_{0}^{2}} \frac{\partial N_{1}^{2}}{\partial z_{*}}-\frac{2 N_{0}^{2}}{S_{0}^{3}} \frac{\partial S_{1}}{\partial z_{*}}\right]
$$

and

$$
\frac{\partial G_{*}}{\partial z_{*}}=\epsilon\left[\frac{1}{\nu N_{0}^{2}} \frac{\partial \varepsilon_{1}}{\partial z_{*}}-\frac{\varepsilon_{0}}{\nu N_{0}^{4}} \frac{\partial N_{1}^{2}}{\partial z_{*}}\right]
$$

while the derivative of $\kappa_{e}$ can be written

$$
\frac{\partial \kappa_{e}}{\partial z_{*}}=\frac{\partial \kappa_{e}}{\partial J_{*}} \frac{\partial J_{*}}{\partial z_{*}}+\frac{\partial \kappa_{e}}{\partial G_{*}} \frac{\partial G_{*}}{\partial z_{*}} .
$$

Using the expansions introduced above, the $O(\epsilon)$ contributions to Eq. 2.3 are

$$
\frac{\partial N_{1}^{2}}{\partial t}=\left[\frac{\partial \kappa_{e}}{\partial J_{*}} \frac{\partial^{2} J_{*}}{\partial z_{*}^{2}}+\frac{\partial \kappa_{e}}{\partial G_{*}} \frac{\partial^{2} G_{*}}{\partial z_{*}^{2}}\right] N_{0}^{2}+\kappa_{e} \frac{\partial^{2} N_{1}^{2}}{\partial z_{*}^{2}},
$$

or using Eqns. 2.9 and 2.10 and their derivatives,

$$
\frac{\partial N_{1}^{2}}{\partial t}=\left[J_{0} \frac{\partial \kappa_{e}}{\partial J_{*}}-G_{0} \frac{\partial \kappa_{e}}{\partial G_{*}}+\kappa_{e}\right] \frac{\partial^{2} N_{1}^{2}}{\partial z_{*}^{2}}-2 S_{0} J_{0}^{2} \frac{\partial^{2} S_{1}}{\partial z_{*}^{2}} \frac{\partial \kappa_{e}}{\partial J_{*}}+\frac{1}{\nu} \frac{\partial^{2} \varepsilon_{1}}{\partial z_{*}^{2}} \frac{\partial \kappa_{e}}{\partial G_{*}} .
$$

The term in brackets on the right hand side of Eq. (2.13) can be interpreted as a diffusivity acting on the sorted buoyancy gradient. When the undisturbed state has

$$
J_{*} \frac{\partial \kappa_{e}}{\partial J_{*}}-G_{*} \frac{\partial \kappa_{e}}{\partial G_{*}}+\kappa_{e}<0
$$

the diffusivity is negative, providing a mechanism for small disturbances to the density gradient to intensify. Since $J_{*}$ and $G_{*}$ are both positive for a stable stratification, conditions are favourable for this mechanism when $\partial \kappa_{e} / \partial J_{*}<0$ and $\partial \kappa_{e} / \partial G_{*}>0$. The 
last two terms in Eq. 2.13 can also influence the evolution of $N_{1}^{2}$. These terms will be relatively small when variations in $J_{*}$ and $G_{*}$ are dominated by changes in $N_{*}^{2}$.

Eqns. 2.3 and 2.14 both contain terms proportional to $\kappa_{e}$ which act against layer formation. Flows with larger values of $\kappa_{e}$ will be less susceptible to layer formation. Molecular diffusion implicitly enters through these terms. Recall that $\kappa_{e}$ cannot be smaller than the molecular diffusivity, $\kappa_{e} \geqslant \kappa$. Therefore, when the molecular diffusivity is large (e.g. when the Prandtl or Schmidt numbers are small), layer formation will be inhibited.

The dependence of the criterion in Eq. 2.14 on molecular diffusion and the Prandtl number can be made more explicit by re-casting the criterion in terms of the irreversible mixing efficiency defined in Peltier \& Caulfield (2003). Salehipour \& Peltier (2015) recently showed that

$$
\frac{\kappa_{e}}{\kappa}=\operatorname{Pr} \Gamma G_{*}+1,
$$

where $\Gamma=E /(1-E)$ is a flux coefficient and $E$ is the irreversible mixing efficiency. Note that we have added laminar diffusion to Eq. 2.15 through the second term on the right hand side. This term was neglected by Salehipour \& Peltier (2015), but is not negligible unless $\Gamma \operatorname{Pr} G_{*}>>1$. If we assume that the irreversible mixing efficiency, $E$, is a function of $J_{*}$ and $G_{*}$, then the condition in Eq. 2.14 can be written

$$
J_{*} \frac{\partial E}{\partial J_{*}}-G_{*} \frac{\partial E}{\partial G_{*}}+\frac{(1-E)^{2}}{\operatorname{Pr} G_{*}}<0
$$

When $\operatorname{Pr} G_{*}=\varepsilon_{*} /\left(\kappa N_{*}^{2}\right)$ is small, the last term on the left hand side of Eq. 2.16 will be large and positive, and will make the condition in Eq. 2.14 more difficult to meet.

We can recover criteria analogous to those obtained by Phillips (1972) and Posmentier (1977) by assuming that $\kappa_{e}$ is independent of $G_{*}$ so that $\partial \kappa_{e} / \partial G_{*}=0$. The last term in Eq. (2.13) is then identically zero. Further neglecting the second to last term in Eq. (2.13), under the assumption that variations in $J_{*}$ are dominated by changes in $N_{*}^{2}$, gives

$$
\frac{\partial N_{1}^{2}}{\partial t} \simeq \frac{\partial^{2} N_{1}^{2}}{\partial z_{*}^{2}} \frac{\partial}{\partial J_{*}}\left(J_{*} \kappa_{e}\right)
$$

When $\partial\left(J_{*} \kappa_{e}\right) / \partial J_{*}<0$, Eq. 2.17 becomes a diffusion equation with a negative diffusion coefficient, and as pointed out by Phillips (1972), small disturbances in the density gradient will grow in time. If the shear rate, $S$, is constant, this condition is also equivalent to $\partial B_{*} / \partial N_{*}^{2}<0$, where $B_{*}=N_{*}^{2} \kappa_{e}$ is the buoyancy flux in sorted buoyancy coordinates. This is the same expression obtained by Posmentier (1977) except that the buoyancy flux is defined in sorted buoyancy coordinates.

Linden (1979) showed that the layer formation condition proposed by Posmentier (1977) can be written $\partial R_{f} / \partial J_{o}<0$, where $R_{f}=B /(B+\varepsilon)$ is the flux Richardson number characterizing turbulent mixing, and $J_{o}$ is an overall Richardson number characterizing the strength of stratification. A similar condition can be obtained as a special case of Eq. 2.16. For $\operatorname{Pr} G_{*}>>1$, the third term on the left hand side of Eq. 2.16 might be negligible. If we can also neglect the second term on the left hand side (e.g. if $E$ is independent of $G_{*}$ ), Eq. 2.16 becomes $\partial E / \partial J_{*}<0$, which is analogous to the condition in Linden (1979) and Posmentier (1977), but now written in terms of the irreversible mixing efficiency, $E$, and the gradient Richardson number defined using sorted buoyancy coordinates, $J_{*}$. 
6

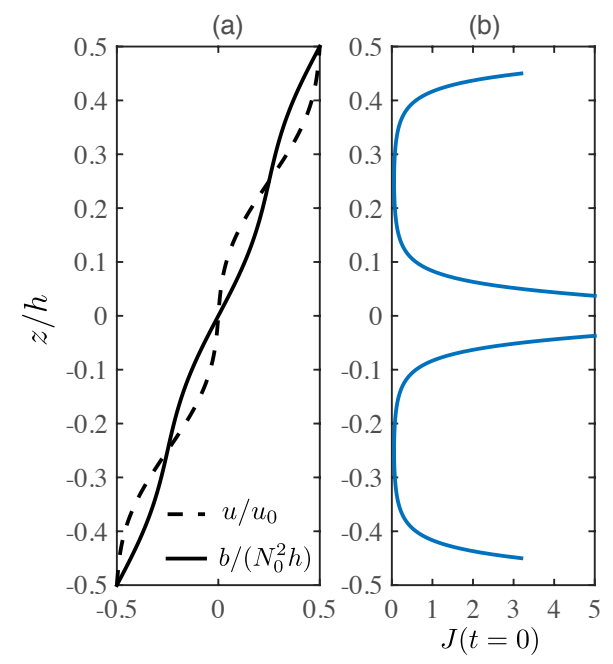

J.R. Taylor, Q. Zhou

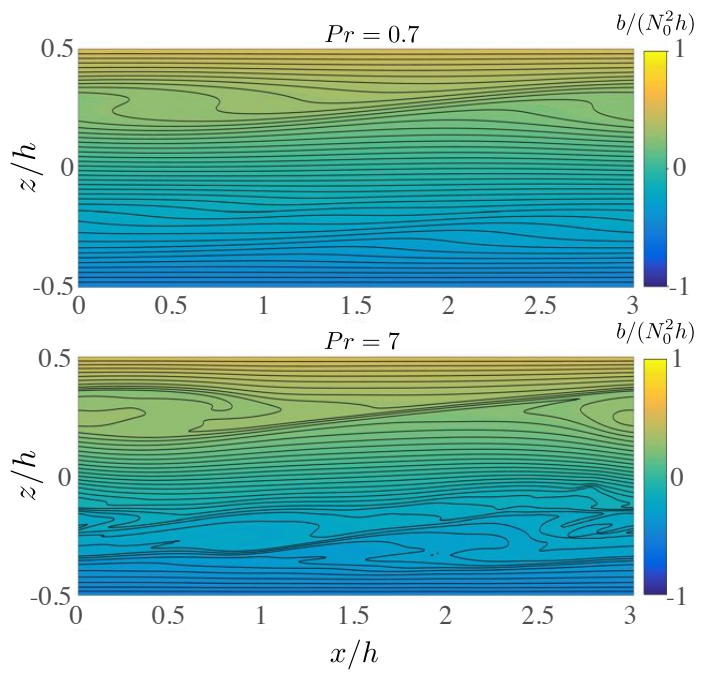

FIGURE 1. Initial mean profiles of buoyancy and velocity used in the DNS (a) and the corresponding gradient Richardson number (b). Vertical $(x-z)$ slices of the buoyancy at time $t \simeq 52 h / u_{0}$ show developing Kelvin-Helmholtz billows and smaller-scale structures in the density field in the case with $\operatorname{Pr}=7$ (bottom) compared with $\operatorname{Pr}=0.7$ (top).

\section{Simulations}

In this section, we apply the framework described above to DNS of a stratified shear flow. The flow is not intended to be typical of the ocean or atmosphere, but rather to demonstrate the possibility of layer formation in a simple configuration. The initial conditions consist of linear velocity and density profiles with sinusoidal disturbances:

$$
\begin{aligned}
& u=S_{0} z-\delta S_{0} \frac{h}{4 \pi} \sin \left(\frac{4 \pi z}{h}\right), \\
& b=N_{0}^{2} z+\gamma N_{0}^{2} \frac{h}{4 \pi} \sin \left(\frac{4 \pi z}{h}\right),
\end{aligned}
$$

where $u$ is the velocity in the $x$ direction and $\delta$ and $\gamma$ are non-dimensional constants that control the relative size of the disturbances to the shear and stratification. The flow is bounded from above and below by rigid horizontal surfaces with boundary conditions $u= \pm u_{0} / 2$ and $b= \pm b_{0} / 2$ at $z= \pm h / 2$ where $u_{0}=S_{0} h$ and $b_{0}=N_{0}^{2} h$. The flow is periodic in the horizontal $(x, y)$ directions and the domain size is $L X=3 h, L Y=0.25 h$. The non-dimensional parameters are $R e \equiv u_{0} h / \nu=14000, J_{0}=N_{0}^{2} / S_{0}^{2}=0.3, \delta=0.8$, and $\gamma=0.4$. Two Prandtl numbers are considered, $\operatorname{Pr}=0.7$ and $\operatorname{Pr}=7$, corresponding to typical values for the diffusion of heat in air and water, respectively.

The incompressible, Boussinesq Navier-Stokes equations are solved using the solver developed by Taylor (2008) and used previously in Deusebio et al. (2015) and Taylor et al. (2016) to study stratified shear flow. A pseudo-spectral method is applied in the horizontal directions and a second-order finite difference method is used for vertical derivatives. The equations are timestepped with mixed implicit/explicit scheme using a third-order accurate Runge-Kutta algorithm and Crank-Nicolson. Further details of the numerical method can be found in Taylor (2008). Both simulations use $384 \times 32 \times 193$ gridpoints, which ensures that the grid spacing is no larger than 2.5 times the Batchelor scale as in the DNS of Smyth \& Moum (2000). To speed up the transition to turbulence, the velocity field was seeded with uniform random noise with an amplitude of $0.2 u_{0}$. The PDF method introduced by Tseng \& Ferziger (2001) was used to calculate the sorted buoyancy profile. 
A multi-parameter criterion for layer formation in a stratified shear flow using sorted buoyancy coordinatē
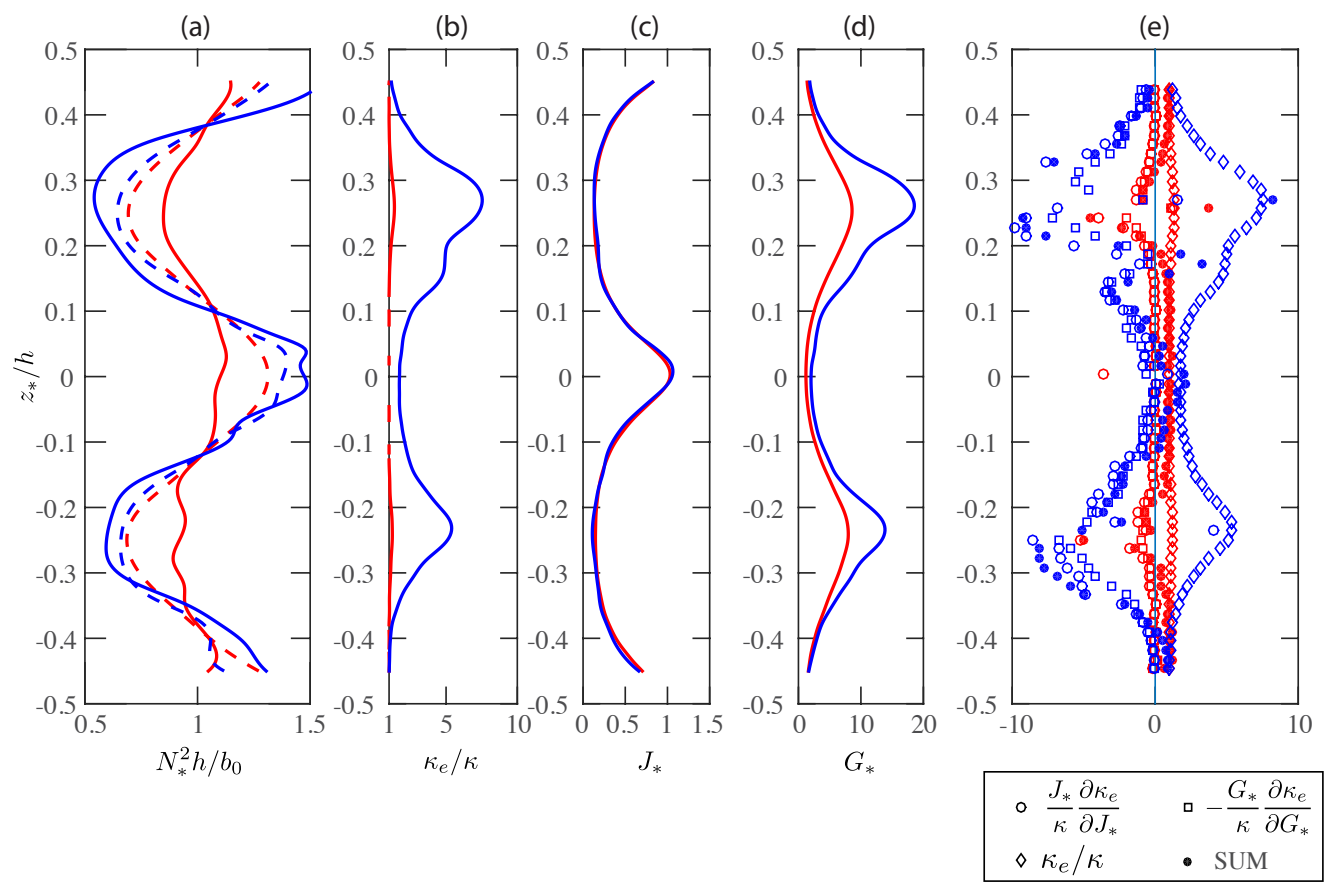

FiguRE 2. Vertical profiles in sorted buoyancy coordinates $\left(z_{*}\right)$ for simulations with $\operatorname{Pr}=0.7$ (red) and $\operatorname{Pr}=7$ (blue). (a) Normalized $N_{*}^{2}$ at $t \simeq 14 h / u_{0}$ (dashed) and $t=105 h / u_{0}$ (solid). (b) Normalized effective diffusivity $\kappa_{e} / \kappa$. (c) Gradient Richardson number, $J_{*}$. (d) buoyancy Reynolds number, $G_{*}$. (e) Terms in the Eq. 2.14, each normalized by $\kappa$.

Initial profiles of velocity and buoyancy are shown in Fig. 1, along with the corresponding gradient Richardson number. Based on the Miles-Howard theorem, the flow has the capacity to support growing linear normal mode perturbations since $J<1 / 4$ in regions above and below the centerline. Overall, the disturbances to the linear velocity and buoyancy profile (and variations in the gradient Richardson number) are large. The framework described above, where small disturbances were considered, can be viewed as an analysis of a narrow range of $z_{*}$. Kelvin-Helmholtz (K-H) billows develop in the weakly stratified regions which distort the buoyancy contours (Fig. 1), with stronger distortion and smaller features in the $\operatorname{Pr}=7$ simulation.

Interestingly, the initial disturbances to the stratification grow over time in the strongly stratified region near the centerline $(z=0)$ for $\operatorname{Pr}=7$ but not when $\operatorname{Pr}=0.7$. Fig. 2(a) shows profiles of $N_{*}^{2}$ for both simulations at $t \simeq 14 h / u_{0}$ after decay of the initial random noise and at $t \simeq 105 h / u_{0}$, corresponding to a time after the first $\mathrm{K}-\mathrm{H}$ mixing event. In the simulation with $\operatorname{Pr}=7$ (blue), the stratification decreases in the regions that had $J<0.25$ at the start of the simulation, while the stratification intensifies near the centerline. In contrast, $N_{*}^{2}$ evolves towards a constant value in the simulation with $\operatorname{Pr}=0.7$ (red).

The amplification of the perturbations to the $N_{*}^{2}$ profile can be analyzed using the theoretical framework introduced above. Profiles of $\kappa_{e}, J_{*}$, and $G_{*}$ at individual times are relatively noisy and intense mixing occurs in intermittent bursts. However, the general trends in each simulation can be seen by examining the time-averaged profiles. Fig. 2 (b) shows profiles of $\kappa_{e}$, normalized by the molecular value, and averaged in the time interval bounded by the profiles shown in Fig. 2(a). The normalized effective diffusivity 
is larger in the case with $\operatorname{Pr}=7$. In both cases, $\partial^{2} \kappa_{e} / \partial z_{*}^{2}<0$ in regions with relatively weak stratification and $\partial^{2} \kappa_{e} / \partial z_{*}^{2}>0$ in regions with relatively strong stratification. This implies that the first term on the right hand side of Eq. 2.3 will tend to reinforce perturbations to the $N_{*}^{2}$ profile. Time-averaged profiles of $J_{*}$ and $G_{*}$ are shown in Figs. $2(\mathrm{c})$ and (d). Regions with large $\kappa_{e}$ are associated with small $J_{*}$ and large $G_{*}$. Here, profiles of $J_{*}\left(z_{*}\right)$ and $G_{*}\left(z_{*}\right)$ are very close to the profiles of the more conventional definitions in terms of unsorted height, $J(z)$ and $G(z)$ (not shown).

The terms in Eq. 2.14 are shown in Fig. 2(e) where each term is evaluated based on the time-averaged profiles of $\kappa_{e}, J_{*}$, and $G_{*}$. Since the initial perturbations in the mean $J_{*}$ and $G_{*}$ profiles are not small as assumed in obtaining Eq. 2.13, we have used the local values of $J_{*}$ and $G_{*}$ in evaluating Eq. 2.14, consistent with the interpretation that the framework applies to a small range of $z_{*}$. The first two terms in Eq. 2.14 are generally negative and hence encourage negative diffusion and layer formation. On the other hand, as noted above, $\kappa_{e}>0$. When $\operatorname{Pr}=0.7$ (red) $\kappa_{e}$ is generally larger than the other terms, resulting in a positive diffusivity in Eq. 2.13. Although $\kappa_{e} / \kappa$ is larger for $\operatorname{Pr}=7$, the other terms more than compensate, and the condition in Eq. 2.14 is met at most locations. These results are consistent with the observation that changes in stratification intensify when $\operatorname{Pr}=7$, but not when $\operatorname{Pr}=0.7$.

Figure 2(e) includes the terms inside the bracket in Eq. 2.13 and hence determines whether the diffusion acting on $N_{1}$ is positive or negative. However, in this particular case, the last two terms in Eq. 2.13 are not negligible. Based on our initial conditions, $\partial^{2} S_{*} / \partial z_{*}^{2}$ has the opposite sign to $\partial^{2} N_{*}^{2} / \partial z_{*}^{2}$. Similarly, as the simulation evolves, $\varepsilon_{*}$ is large where $N_{*}^{2}$ is small and although there are large fluctuations $\partial^{2} \varepsilon_{*} / \partial z_{*}^{2}$ generally has the opposite sign to $\partial^{2} N_{*}^{2} / \partial z_{*}^{2}$. As a result, both terms encourage layer formation in our simulations.

\section{Parameterizations}

The previous section demonstrates that the framework developed in section 2 is useful for diagnosing the sharpening of a density profile though stratified turbulence. The framework can be applied more broadly by assuming a particular functional form for $\kappa_{e}\left(J_{*}, G_{*}\right)$. A simple expression follows if we assume that $\kappa_{e}$ depends on $J_{*}$ and $G_{*}$ through a power law:

$$
\frac{\kappa_{e}}{\kappa}=J_{*}^{\alpha} G_{*}^{\beta} f(\operatorname{Pr}, \ldots),
$$

where $f(\operatorname{Pr}, \ldots)$ includes the explicit dependence of $\kappa_{e}$ on the Prandtl number and any other constant parameters. This form is unlikely to hold over a wide range of $J_{*}$ and $G_{*}$ for fixed values of $\alpha$ and $\beta$, but it might provide a reasonable approximation to $\kappa_{e}$ for a limited range of $J_{*}$ and $G_{*}$. The condition for a negative diffusivity in Eq. 2.14 then becomes simply

$$
\alpha-\beta+1<0 .
$$

Based on DNS of homogeneous sheared stratified turbulence, Shih et al. (2005) found that the turbulent diffusivity increased with increasing $G$ with an exponent of $\beta \simeq 1$ for $7<G<100$ and $\beta \simeq 0.5$ for $G>100 \dagger$. Without additional dependence on the gradient Richardson number, this value of $\beta$ would not be sufficient for Eq. 2.14 to hold.

Recently, Salehipour et al. (2016) collected data from DNS and developed a parameter-

$\dagger$ Note that the turbulent diffusivity in Shih et al. (2005) is defined in terms of the buoyancy flux and buoyancy gradient in the vertical $(z)$ direction, while all quantities here are defined in terms of derivatives in sorted buoyancy coordinates, $z_{*}$. 


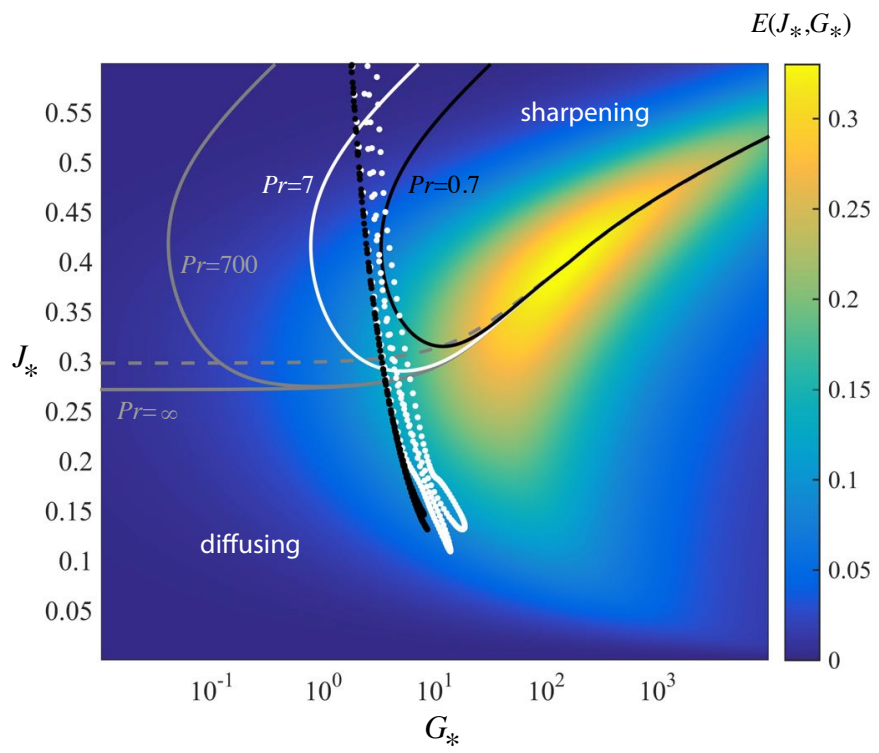

FiguRE 3. Irreversible mixing efficiency, $E\left(J_{*}, G_{*}\right)$, as parameterized by Salehipour et al. (2016) (colour shading). Solid contours separate regions where the bracketed term in Eq. 2.13 is sharpening and diffusive for four different values of the Prandtl (or Schmidt) number, $\operatorname{Pr}=\nu / \kappa$. The dashed line delineates the region where $\partial E / \partial J_{*}=0$. Mean profiles of $J_{*}$ and $G_{*}$ from the DNS (as plotted in Figure 2) are shown using white dots for $\operatorname{Pr}=7$ and black dots for $\operatorname{Pr}=0.7$.

ization for the irreversible mixing efficiency, $E$, as a function of the gradient Richardson and buoyancy Reynolds numbers in sorted buoyancy coordinates, $J_{*}$ and $G_{*}$. Details of this parameterization can be found in Salehipour et al. (2016). The color shading in Fig. 3 shows the parameterized form of $E\left(G_{*}, J_{*}\right)$, which notably has non-monotonic behavior in both parameters. Solid contours separate regions where the condition in Eq. 2.16 holds for three different values of the Prandtl (or Schmidt) number, $\operatorname{Pr}=0.7,7,700$, corresponding approximately to the diffusion of heat in air, heat in water, and salt in water. For reference, a line showing $\operatorname{Pr}=\infty$ is also shown. There is non-trivial Prandtl and buoyancy Reynolds dependence on the condition in Eq. 2.16 with the parameterized form of $E\left(G_{*}, J_{*}\right)$. The Prandtl number has a strong effect on the boundary for the range $J_{*} \gtrsim 0.25$ and $G_{*} \lesssim 100$. Salehipour et al. (2016) note that $E$ might depend explicitly on the Prandtl number, although they didn't include this dependence in their parameterization.

The dashed line in Fig. 3 indicates where $\partial E / \partial J_{*}=0$, and the region above this line satisfies $\partial E / \partial J_{*}<0$. This gives a very good approximation to the full condition in Eq. 2.16 for $G_{*}>100$. Note, however, that the parameterized form of $E$ and the location where $\partial E / \partial J_{*}=0$ depends strongly on $G_{*}$ in this range.

The DNS results described in section 3 are qualitatively consistent with the sharpening criteria derived from the Salehipour et al. (2016) parameterization. Black and white dots in Fig. 3 show individual points along the mean $J_{*}$ and $G_{*}$ profiles for $\operatorname{Pr}=0.7$ (black) and $\operatorname{Pr}=7$ (white). When $\operatorname{Pr}=0.7$ the values of $J_{*}$ and $G_{*}$ are always in the diffusing regime, while when $\operatorname{Pr}=7$ the profiles pass into the sharpening regime. 


\section{Discussion}

In this paper we revisited the thought experiment proposed by Phillips (1972) and the possibility that small perturbations to a uniform stable density stratification can grow in time, possibly leading to the formation of step-like layers. This extended previous work in two ways. First, the evolution equation for the stratification was written in isoscalar coordinates using the framework introduced by Winters \& D'Asaro (1996) and Nakamura (1996). This has the advantage of removing reversible contributions to the buoyancy flux and provides a useful diagnostic framework for identifying sharpening of stratified interfaces. There is a possibility for an increase in stratification when the curvature of the effective diffusivity in sorted buoyancy coordinates is positive $\left(\partial^{2} \kappa_{e} / \partial z_{*}^{2}>0\right)$ and sufficiently large to overcome diffusion by $\kappa_{e}$.

We then assumed that the effective diffusivity could be written as a function of the gradient Richardson number, $J_{*}$, and buoyancy Reynolds number, $G_{*}$, defined in terms of quantities averaged in sorted buoyancy coordinates. Using this framework, small departures to a constant stratification can be written in the form of a diffusion equation with two additional terms that depend on variations in the background shear and turbulent dissipation rate. The coefficient of diffusion is negative when the effective diffusivity decreases with increasing gradient Richardson number and/or increases with increasing buoyancy Reynolds number, such that these changes overcome the stabilizing influence of diffusion through $\kappa_{e}$. This criterion was also re-written in terms of the irreversible mixing efficiency in Eq. 2.16 using a relation recently derived by Salehipour \& Peltier (2015).

DNS of a stratified shear flow demonstrate that existing disturbances to an otherwise linear density profile can grow following a mixing event. This occurred when the molecular Prandtl number was 7 (appropriate for the diffusion of heat in water), but not when the Prandtl number was 0.7 (appropriate for the diffusion of heat in air). Since the simulations used a linear equation of state, the simulations exclude the possible role of double diffusion. Instead, the tendency for the density profile to sharpen is a direct result of stratified turbulence.

The sharpening of the buoyancy profile seen in the DNS with $\operatorname{Pr}=7$ is small and transient. It is possible that a more dramatic sharpening would be seen for $\operatorname{Pr}=700$, although this is presently beyond the reach of DNS. Further sharpening could develop in response to a series of mixing events, each similar to the one described in section 3. For example, if a train of low frequency internal gravity waves propagated through a pre-existing shear layer, the oscillating shear associated with the internal waves could trigger repeated $\mathrm{K}-\mathrm{H}$ mixing events similar to the one simulated here, and the net result might be the development of a step-like density profile.

Although Eq. 2.14 doesn't depend explicitly on the Prandtl number (or any other constant non-dimensional parameters), the Prandtl number can influence this condition in two ways. First, since $\kappa_{e}$ is bounded from below by $\kappa$, more diffusive substances with small Prandtl number could lead to larger values of $\kappa_{e}$, making it more difficult for Eq. 2.14 to be met. Second, the DNS results show that the Prandtl number can also change the dependence of $\kappa_{e}$ on $J_{*}$ and $G_{*}$. The first two terms in Eq. 2.14 are more negative for $\operatorname{Pr}=7$ than for $\operatorname{Pr}=0.7$, and this is true even when the size of the terms is not scaled with $\kappa$ (not shown).

In order to evaluate the condition for sharpening through negative diffusion in Eq. 2.14 , the functional dependence of $\kappa_{e}$ on $J_{*}, G_{*}$, and $\operatorname{Pr}$ (and possibly other parameters) must be specified. Using a parameterization recently developed by Salehipour et al. (2016), Eq. 2.16 yields a criterion that depends non-trivially on $J_{*}, G_{*}$, and $\operatorname{Pr}$, but 
sharpening through negative diffusion generally occurs when $J_{*}$ and $G_{*}$ are large, and positive diffusion occurs more readily when the Prandtl number is small. Note that the simulations used by Salehipour et al. (2016) as a basis for their parameterization didn't cover the full range of $J_{*}$ and $G_{*}$ shown in Fig. 3, and the details of the criterion should be viewed with caution. The criteria for sharpening through negative diffusion shown in Fig. 3 is specific to the parameterization developed by Salehipour et al. (2016), although the framework developed here could be applied more generally.

The formation of a step-like density profile has been seen in several of laboratory experiments where grids or bars were towed through a fluid with an initially linear density profile (Ruddick et al. 1989; Holford \& Linden 1999; Park et al. 1994). As recently reviewed by Thorpe (2016), regimes with and without layer formation are separated by a boundary which depends on the Reynolds and Froude numbers associated with the towed grid. Different studies have proposed different functional forms for this boundary, and the estimates are not always consistent. Generally layers are not seen when the Froude number is too large, and layer formation appears to be favored for moderate Reynolds numbers. The framework presented here could be used to analyze layer formation in these experiments. For example, the analysis in section 2 could be repeated to include dependence of $\kappa_{e}$ on a turbulent Froude number associated with an oscillating grid.

In the thought experiment presented by Phillips (1972), a vertical buoyancy flux was maintained by turbulence which was modulated by changes to the stratification. The derivation above raises the possibility that internal waves might be capable of inducing layer development without requiring turbulence or density overturns. This does not contradict the formulation in tracer coordinates which excludes reversible buoyancy fluxes. Instead, if the displacement of isopycnals is persistently large in regions of weak stratification, an increase in the tracer area and the effective diffusivity in the region of weak stratification could lead to layer formation. Perhaps consistent with this hypothesis, Fig. 3 suggests that when $\operatorname{Pr}=700$, layer formation might be supported for $G_{*}<1$, in a part of parameter space where stratification effects are felt down to the viscous scale.

The EPSRC Programme Grant EP/K034529/1 entitled 'Mathematical Underpinnings of Stratified Turbulence' (MUST) is gratefully acknowledged for supporting the research presented here. We are grateful to the MUST team for many helpful discussions and suggestions and to two anonymous referees whose comments helped improve the manuscript. Supporting data in the form of source code and input files for the direct numerical simulations can be obtained from https://doi.org/10.17863/CAM.9631.

\section{REFERENCES}

Bartello, P \& Tobias, SM 2013 Sensitivity of stratified turbulence to the buoyancy reynolds number. Journal of Fluid Mechanics 725, 1-22.

Bouffard, D \& Boegman, L 2013 A diapycnal diffusivity model for stratified environmental flows. Dynamics of Atmospheres and Oceans 61, 14-34.

Brethouwer, G, Billant, P, Lindborg, E \& Chomaz, J-M 2007 Scaling analysis and simulation of strongly stratified turbulent flows. Journal of Fluid Mechanics 585, 343-368.

Deusebio, E, Caulfield, CP \& TAYlor, JR 2015 The intermittency boundary in stratified plane couette flow. Journal of Fluid Mechanics 781, 298-329.

Dillon, Thomas M \& Caldwell, Douglas R 1980 The batchelor spectrum and dissipation in the upper ocean. Journal of Geophysical Research: Oceans 85 (C4), 1910-1916.

Gargett, AE, Osborn, TR \& NAsmyth, PW 1984 Local isotropy and the decay of turbulence in a stratified fluid. Journal of Fluid Mechanics 144, 231-280. 
GarRetT, C \& Munk, W 1971 Internal wave spectra in the presence of fine-structure. Journal of Physical Oceanography 1 (3), 196-202.

Gibson, CH 1980 Fossil temperature, salinity, and vorticity turbulence in the ocean. Elsevier Oceanography Series 28, 221-257.

Holford, JM \& Linden, PF 1999 Turbulent mixing in a stratified fluid. Dynamics of atmospheres and oceans 30 (2), 173-198.

Linden, PF 1979 Mixing in stratified fluids. Geophysical $\&$ Astrophysical Fluid Dynamics 13 (1), $3-23$.

Linden, PF 1980 Mixing across a density interface produced by grid turbulence. Journal of Fluid Mechanics 100 (04), 691-703.

Lozovatsky, ID \& Fernando, HJS 2013 Mixing efficiency in natural flows. Philosophical Transactions of the Royal Society of London A: Mathematical, Physical and Engineering Sciences 371 (1982), 20120213.

Mater, BD \& Venayagamoorthy, SK 2014a The quest for an unambiguous parameterization of mixing efficiency in stably stratified geophysical flows. Geophysical Research Letters 41 (13), 4646-4653.

Mater, BD \& Venayagamoorthy, SK $2014 b$ A unifying framework for parameterizing stably stratified shear-flow turbulence. Physics of Fluids (1994-present) 26 (3), 036601.

NAKAMURA, N 1996 Two-dimensional mixing, edge formation, and permeability diagnosed in an area coordinate. Journal of the atmospheric sciences 53 (11), 1524-1537.

Park, Y-G, Whitehead, JA \& Gnanadeskian, A 1994 Turbulent mixing in stratified fluids: layer formation and energetics. Journal of Fluid Mechanics 279, 279-311.

Pelegrí, JL \& SANGRÀ, P 1998 A mechanism for layer formation in stratified geophysical flows. Journal of Geophysical Research: Oceans 103 (C13), 30679-30693.

Peltier, WR \& Caulfield, CP 2003 Mixing efficiency in stratified shear flows. Annual review of fluid mechanics 35 (1), 135-167.

Phillips, OM 1972 Turbulence in a strongly stratified fluid-is it unstable? In Deep Sea Research and Oceanographic Abstracts, , vol. 19, pp. 79-81. Elsevier.

Portwood, G., de Bruyn Kops S., Taylor, J.R., Salehipour, H. \& Caulfield, C.P. 2016 Robust identification of dynamically distinct regions in stratified turbulence. Journal Fluid Mechanics Rapids in press.

Posmentier, ES 1977 The generation of salinity finestructure by vertical diffusion. Journal of Physical Oceanography 7 (2), 298-300.

RAdKo, Timour 2016 Thermohaline layering in dynamically and diffusively stable shear flows. Journal of Fluid Mechanics 805, 147-170.

Ruddick, BR, McDougall, TJ \& Turner, JS 1989 The formation of layers in a uniformly stirred density gradient. Deep Sea Research Part A. Oceanographic Research Papers 36 (4), 597-609.

Salehipour, H \& Peltier, WR 2015 Diapycnal diffusivity, turbulent prandtl number and mixing efficiency in boussinesq stratified turbulence. Journal of Fluid Mechanics 775, 464500 .

Salehipour, H, Peltier, WR, Whalen, CB \& MacKinnon, JA 2016 A new characterization of the turbulent diapycnal diffusivities of mass and momentum in the ocean. Geophysical Research Letters 43 (7), 3370-3379.

Schмiтt, RW 1994 Double diffusion in oceanography. Annual Review of Fluid Mechanics 26 (1), 255-285.

Shin, LH, Koseff, JR, IVey, GN \& Ferziger, JH 2005 Parameterization of turbulent fluxes and scales using homogeneous sheared stably stratified turbulence simulations. Journal of Fluid Mechanics 525, 193-214.

Sмүтн, WD \& Moum, JN 2000 Length scales of turbulence in stably stratified mixing layers. Physics of Fluids 12 (6), 1327-1342.

TAYLOR, JR 2008 Numerical simulations of the stratified oceanic bottom boundary layer. University of California, San Diego.

Taylor, JR, Deusebio, E, Caulfield, CP \& Kerswell, RR 2016 A new method for isolating turbulent states in transitional stratified plane couette flow. Journal of Fluid Mechanics 808 (R1). 
A multi-parameter criterion for layer formation in a stratified shear flow using sorted buoyancy coordinatle3

THORPE, SA 2016 Layers and internal waves in uniformly stratified fluids stirred by vertical grids. Journal of Fluid Mechanics 793, 380-413.

Tseng, Y. \& Ferziger, J. H. 2001 Mixing and available potential energy in stratified flows. Phys. Fluids 13, 1281-1293.

Winters, KB \& D'Asaro, EA 1996 Diascalar flux and the rate of fluid mixing. Journal of Fluid Mechanics 317, 179-193.

Zhou, Q, Taylor, JR, Caulfield, CP \& Linden, PF 2017 Diapycnal mixing in layered stratified plane couette flow quantified in a tracer-based coordinate. Journal of Fluid Mechanics in press. 